Article

\title{
The Bregman-Opial Property and Bregman Generalized Hybrid Maps of Reflexive Banach Spaces
}

\author{
Eskandar Naraghirad ${ }^{1, *}$, Luoyi Shi ${ }^{2}$ and Ngai-Ching Wong ${ }^{3}$ (1) \\ 1 Department of Mathematics, Yasouj University, Yasouj 75918, Iran \\ 2 School of Mathematical Sciences, Tiangong University, Tianjin 300387, China; shiluoyitjpu@163.com \\ 3 Department of Applied Mathematics, National Sun Yat-sen University, Kaohsiung 80424, Taiwan; \\ wong@math.nsysu.edu.tw \\ * Correspondence: eskandarrad@gmail.com or esnaraghirad@yu.ac.ir
}

Received: 10 May 2020; Accepted: 19 June 2020; Published: 22 June 2020

\begin{abstract}
The Opial property of Hilbert spaces is essential in many fixed point theorems of non-expansive maps. While the Opial property does not hold in every Banach space, the Bregman-Opial property does. This suggests to study fixed point theorems for various Bregman non-expansive like maps in the general Banach space setting. In this paper, after introducing the notion of Bregman generalized hybrid sequences in a reflexive Banach space, we prove (with using the Bregman-Opial property instead of the Opial property) convergence theorems for such sequences. We also provide new fixed point theorems for Bregman generalized hybrid maps defined on an arbitrary but not necessarily convex subset of a reflexive Banach space. We end this paper with a brief discussion of the existence of Bregman absolute fixed points of such maps.
\end{abstract}

Keywords: Bregman-Opial property; Bregman generalized hybrid map/sequence; Bregman absolute fixed point; convergence theorem; fixed point theorem

MSC: $46 S 40$

\section{Introduction}

Let $T: C \rightarrow E$ be a nonexpansive map from a nonempty subset $C$ of a (real) Banach space $E$ into $E$. Several iterative schemes, e.g., in [1-3], developed for locating fixed points in $F(T)=\{x \in C: T x=x\}$ assume the Opial property [4] of $E$. The Opial property states that for any weakly convergent sequence $x_{n} \rightarrow x$ in $E$, we have

$$
\limsup _{n \rightarrow \infty}\left\|x_{n}-x\right\|<\limsup _{n \rightarrow \infty}\left\|x_{n}-y\right\|, \quad \text { for all } y \in E \backslash\{x\} .
$$

It is well known that all Hilbert spaces, all finite dimensional Banach spaces, and the Banach spaces $l^{p}(1 \leq p<\infty)$ satisfy the Opial property. However, not every Banach space satisfies the Opial property; see, for example, $[5,6]$. We thus ask for a more subtle property to implement with the general iterative fixed point algorithms.

The Bregman distance $D_{g}$ is an appropriate candidate, because it holds the Bregman-Opial inequality for any Banach space as shown in Lemma 1 below. Let $g: E \rightarrow \mathbb{R}$ be a strictly convex and Gâteaux differentiable function on a Banach space $E$. The Bregman distance [7] (see also [8,9]) $D_{g}$ on $E$ is defined by

$$
D_{g}(x, y)=g(x)-g(y)-\langle x-y, \nabla g(y)\rangle, \quad \text { for all } x, y \in E .
$$


It follows from the strict convexity of $g$ that $D_{g}(x, y) \geq 0$ for all $x, y$ in $E$; and $D_{g}(x, y)=0$ exactly when $x=y$. However, $D_{g}$ might not be symmetric and $D_{g}$ might not satisfy the triangular inequality.

Lemma 1 (([10], Lemma 5.1), see also [11]). Let $g: E \rightarrow \mathbb{R}$ be a strictly convex and Gâteaux differentiable function on a Banach space $E$. For any weakly convergent sequence $x_{n} \rightarrow x$ in $E$, we have

$$
\limsup _{n \rightarrow \infty} D_{g}\left(x_{n}, x\right)<\limsup _{n \rightarrow \infty} D_{g}\left(x_{n}, y\right), \quad \text { for all } y \in E \backslash\{x\} .
$$

As shown in the proof of ([10], Lemma 5.1), an alternative form of the Bregman-Opial property reads

$$
\liminf _{n \rightarrow \infty} D_{g}\left(x_{n}, x\right)<\liminf _{n \rightarrow \infty} D_{g}\left(x_{n}, y\right), \quad \text { for all } y \in E \backslash\{x\} .
$$

When $E$ is a smooth Banach space, if we choose the Bregman function $g(x)=\|x\|^{2}$ then $\nabla g(x)=$ $2 J x$, where $J$ is the normalized duality mapping from $E$ into its Banach dual space $E^{*}$. The Bregman distance $D_{g}(\cdot, \cdot)$ reduces to the usual bilinear form $\phi(\cdot, \cdot)$ as

$$
D_{g}(x, y)=\phi(x, y):=\|x\|^{2}-2\langle x, J y\rangle+\|y\|^{2}, \quad \text { for all } x, y \in E .
$$

In particular, when $E$ is a Hilbert space, we have $D_{g}(x, y)=\|x-y\|^{2}$.

The Bregman distance $D_{g}$ is widely used in quantum information theory. Let $f:[0,+\infty) \rightarrow \mathbb{R}$ be any strictly convex and Fréchet differentiable function. When $g=$ trace $\circ f$, it arises from (1) the Bregman divergence between quantum data, i.e., positive-definite square matrices,

$$
D_{g}(A, B)=\operatorname{trace}\left(f(A)-f(B)-f^{\prime}(B)(A-B)\right) .
$$

Here, the matrices $f(A), f(B)$ and $f^{\prime}(B)$ are defined through functional calculus. For example, we have classical divergence $D_{g}(A, B)=\operatorname{trace}\left(A^{2}\right)+\operatorname{trace}\left(B^{2}\right)-2 \operatorname{trace}(B A)$, while $f(x)=x^{2}$, Umegaki relative entropy $D_{g}(A, B)=\operatorname{trace}(A(\log A-\log B))$, while $f(x)=x \log x$, Tsallis relative entropy $D_{g}(A, B)=\frac{1}{q-1} \operatorname{trace}\left(A^{q} B^{1-q}-A\right)$, while $f(x)=\frac{x^{q}-x}{q-1}$, and quantum divergence $D_{g}(A, B)=\|\sqrt{A}-\sqrt{B}\|_{2}^{2}$, while $f(x)=(\sqrt{x}-1)^{2}$.

Here, $\|\cdot\|_{2}$ is the Hilbert-Schmidt norm of matrices. See, e.g., [12] for details.

Let $g: E \rightarrow \mathbb{R}$ be strictly convex and Gâteaux differentiable, and $C \subseteq E$ be nonempty. A mapping $T: C \rightarrow E$ is said to be

- Bregman nonexpansive if

$$
D_{g}(T x, T y) \leq D_{g}(x, y), \quad \text { for all } x, y \in C ;
$$

- Bregman quasi-nonexpansive if the fixed point set $F(T) \neq \varnothing$ and

$$
D_{g}(p, T x) \leq D_{g}(p, x), \quad \text { for all } x \in C, p \in F(T) ;
$$

- Bregman nonspreading if

$$
D_{g}(T x, T y)+D_{g}(T y, T x) \leq D_{g}(T x, y)+D_{g}(T y, x), \quad \text { for all } x, y \in C ;
$$

- Bregman generalized hybrid if there exist $\alpha, \beta \in \mathbb{R}$ such that

$$
\alpha D_{g}(T x, T y)+(1-\alpha) D_{g}(x, T y) \leq \beta D_{g}(T x, y)+(1-\beta) D_{g}(x, y), \quad \text { for all } x, y \in C .
$$

It is clear that nonexpansive, quasi-nonexpansive, nonspreading [13-15], and generalized hybrid [16] maps of Hilbert spaces are exactly those Bregman nonexpansive, Bregman quasi-nonexpansive, Bregman 
nonspreading, and Bregman generalized hybrid maps with respect to the Bregman distance $D_{g}$ with $g(x)=\|x\|^{2}$. Bregman generalized hybrid maps are introduced and studied in [17], and it seems to be one of the most general notions among those mentioned above. We continue to study it in this paper.

The Bregman-Opial property (Lemma 1) suggests the following.

Problem 1. Can we develop fixed point theorems and convergence results for the Picard and other iteration schemes for various Bregman nonexpansive-like maps in the general Banach space setting?

On the other hand, the theory of approximating fixed points of general nonlinear maps has many important applications (see, for example, [18-20]). However, a little work has been done without the convexity assumption. Djafari Rouhani ([21-28]) developed a theory of approximating fixed points for nonlinear maps with non-convex domains in the Hilbert space setting. The Opial property of the underlying Hilbert space plays an important role in Rouhani's theory. This suggests to us to pose another question.

Problem 2. Can we extend fixed point theorems for nonlinear maps on non-convex domains in Hilbert spaces to the more general Banach space setting without assuming the Opial property?

We answer the above questions in this paper. In Section 2, we collect some basic properties of Bregman distances. In Section 3, utilizing the Bregman-Opial property, we investigate the weak convergence of Bregman generalized hybrid sequences, which can be produced by the Picard iterations for Bregman generalized hybrid maps. In Section 4, assuming the existence of a bounded and weakly asymptotically regular orbit, we present fixed point and convergence theorems for Bregman generalized hybrid maps, which might be defined on non-convex domains in reflexive Banach spaces. Finally, in Section 5, we study the existence of absolute fixed points for Bregman generalized hybrid maps.

Our results improve and supplement those in [17], and also some known results in the literature, e.g., $[18,21-33]$.

\section{Preliminaries and Bregman Distances}

Let $E$ be a (real) Banach space with norm $\|\cdot\|$ and dual space $E^{*}$. For any $x$ in $E$, we denote the value of $x^{*}$ in $E^{*}$ at $x$ by $\left\langle x, x^{*}\right\rangle$. When $\left\{x_{n}\right\}_{n \in \mathbb{N}}$ is a sequence in $E$, we denote the strong convergence of $\left\{x_{n}\right\}_{n \in \mathbb{N}}$ to $x \in E$ by $x_{n} \rightarrow x$ and the weak convergence by $x_{n} \rightarrow x$. A bounded sequence $\left\{x_{n}\right\}_{n \in \mathbb{N} \cup\{0\}}$ is said to be asymptotically regular (resp. weakly asymptotically regular), if $x_{n+1}-x_{n} \rightarrow 0$ (resp. $x_{n+1}-x_{n} \rightarrow 0$ ) as $n \rightarrow \infty$.

For any $r>0$, let $B_{r}:=\{z \in E:\|z\| \leq r\}$. A function $g: E \rightarrow \mathbb{R}$ is said to be

- strictly convex if

$$
g(\alpha x+(1-\alpha) y)<\alpha g(x)+(1-\alpha) g(y), \quad \forall \text { distinct } x, y \in E, \forall \alpha \in(0,1) ;
$$

- $\quad$ strongly coercive if

$$
\lim _{\left\|x_{n}\right\| \rightarrow+\infty} \frac{g\left(x_{n}\right)}{\left\|x_{n}\right\|}=+\infty
$$

- locally bounded if $g\left(B_{r}\right)$ is bounded for all $r>0$.

A function $g: E \rightarrow \mathbb{R}$ is said to be Gâteaux differentiable at $x$ if $\lim _{t \rightarrow 0} \frac{g(x+t y)-g(x)}{t}$ exists for any $y$. In this case, the gradient $\nabla g(x)$ is defined as the linear functional in $E^{*}$ such that

$$
\langle y, \nabla g(x)\rangle=\lim _{t \rightarrow 0} \frac{g(x+t y)-g(x)}{t}, \quad \text { for all } y \in E .
$$


We call $g$ Fréchet differentiable at $x$ (see, for example, ([34], p. 13) or ([35], p. 508)) if for all $\epsilon>0$, there exists $\delta>0$ such that

$$
|g(y)-g(x)-\langle y-x, \nabla g(x)\rangle| \leq \epsilon\|y-x\| \quad \text { whenever }\|y-x\| \leq \delta .
$$

The function $g$ is said to be Gâteaux (resp. Fréchet) differentiable if it is Gâteaux (resp. Fréchet) differentiable everywhere. If a convex function $g: E \rightarrow \mathbb{R}$ is Gâteaux differentiable, then $\nabla g$ is norm-to-weak* continuous (see, for example, ([34], Proposition 1.1.10)); if $g$ is Fréchet differentiable, then $\nabla g$ is norm-to-norm continuous (see, ([35], p. 508)).

Let $g: E \rightarrow \mathbb{R}$ be a strictly convex and Gâteaux differentiable function. The Bregman distance defined in (1) satisfies the three-point identity [7]

$$
D_{g}(x, z)=D_{g}(x, y)+D_{g}(y, z)+\langle x-y, \nabla g(y)-\nabla g(z)\rangle, \quad \text { for all } x, y, z \in E .
$$

In particular,

$$
D_{g}(x, y)=-D_{g}(y, x)+\langle y-x, \nabla g(y)-\nabla g(x)\rangle, \quad \text { for all } x, y \in E \text {. }
$$

If $g$ is locally bounded, by Definition (1) we have $\left\{D_{g}(x, y): x \in B_{r}\right\}$ is bounded for all $r>0$.

Let $C$ be a nonempty, closed, and convex subset of $E$ and $\left\{x_{n}\right\}_{n \in \mathbb{N}}$ be a bounded sequence in $E$. For any $x$ in $E$, we set

$$
\operatorname{Br}\left(x,\left\{x_{n}\right\}_{n \in \mathbb{N}}\right)=\limsup _{n \rightarrow \infty} D_{g}\left(x_{n}, x\right) .
$$

The Bregman asymptotic radius of $\left\{x_{n}\right\}_{n \in \mathbb{N}}$ relative to $C$ is defined by

$$
\operatorname{Br}\left(C,\left\{x_{n}\right\}_{n \in \mathbb{N}}\right)=\inf \left\{\operatorname{Br}\left(x,\left\{x_{n}\right\}\right): x \in C\right\} .
$$

The Bregman asymptotic center of $\left\{x_{n}\right\}_{n \in \mathbb{N}}$ relative to $C$ is the set

$$
\operatorname{BAC}\left(C,\left\{x_{n}\right\}_{n \in \mathbb{N}}\right)=\left\{x \in C: \operatorname{Br}\left(x,\left\{x_{n}\right\}_{n \in \mathbb{N}}\right)=\operatorname{Br}\left(C,\left\{x_{n}\right\}_{n \in \mathbb{N}}\right)\right\} .
$$

We call a point in $\operatorname{BAC}\left(E,\left\{x_{n}\right\}_{n \in \mathbb{N}}\right)$ simply a Bregman asymptotic center of $\left\{x_{n}\right\}_{n \in \mathbb{N}}$.

Proposition 1 ([11], Proposition 9). Let $C$ be a nonempty, closed, and convex subset of a reflexive Banach space $E$, and let $g: E \rightarrow \mathbb{R}$ be strictly convex, Gâteaux differentiable, and locally bounded on $E$. If $\left\{x_{n}\right\}_{n \in \mathbb{N}}$ is a bounded sequence of $C$, then $\operatorname{BAC}\left(C,\left\{x_{n}\right\}_{n \in \mathbb{N}}\right)$ is a singleton.

Definition 1. Let $E$ be a Banach space. A function $g: E \rightarrow \mathbb{R}$ is said to be a Bregman function [34] if the following conditions are satisfied:

(i) $g$ is continuous, strictly convex and Gâteaux differentiable;

(ii) the set $\left\{y \in E: D_{g}(x, y) \leq r\right\}$ is bounded for all $x$ in $E$ and $r>0$.

We call $g$ a nice Bregman function if it holds, in addition,

(iii) $g$ is strong coercive, locally bounded, and $\nabla g: E \rightarrow E^{*}$ is weak-to-weak* sequentially continuous.

The following lemma follows from Butnariu and Iusem [34] and Zălinescu [36].

Lemma 2 ([34,36]). Let $E$ be a reflexive Banach space and $g: E \rightarrow \mathbb{R}$ a strongly coercive Bregman function. Then

(i) $\nabla g: E \rightarrow E^{*}$ is one-to-one, onto and norm-to-weak ${ }^{*}$ continuous;

(ii) $\langle x-y, \nabla g(x)-\nabla g(y)\rangle=0$ if and only if $x=y$;

(iii) $\quad\left\{x \in E: D_{g}(x, y) \leq r\right\}$ is bounded for all $y$ in $E$ and $r>0$; 


\section{Bregman Generalized Hybrid Sequences}

We define a new concept of Bregman generalized hybrid sequences which extends the notions of hybrid and nonexpansive sequences introduced and studied in [27].

Definition 2. Fix a Bregman function $g: E \rightarrow \mathbb{R}$ on a reflexive Banach space $E$. A sequence $\left\{x_{n}\right\}_{n \in \mathbb{N} \cup\{0\}}$ in $E$ is said to be a Bregman generalized hybrid sequence if there exist real numbers $\alpha, \beta$ such that

$$
\alpha D_{g}\left(x_{i+1}, x_{j+1}\right)+(1-\alpha) D_{g}\left(x_{i}, x_{j+1}\right) \leq \beta D_{g}\left(x_{i+1}, x_{j}\right)+(1-\beta) D_{g}\left(x_{i}, x_{j}\right), \quad \text { for all } i, j \geq 0 .
$$

It is plain that if $T$ is a Bregman generalized hybrid map then any orbit $\left\{x_{n}:=T^{n} x\right\}_{n \in \mathbb{N} \cup\{0\}}$ is a Bregman generalized hybrid sequence. Here, $x_{0}=T^{0} x=x$ by convention.

Notations 1. Let $E$ be a reflexive Banach space and $g: E \rightarrow \mathbb{R}$ be strictly convex and Gâteaux differentiable on E. Given a sequence $\left\{x_{n}\right\}_{n \in \mathbb{N} \cup\{0\}}$ in E. Denote by

$$
\begin{aligned}
s_{n} & :=\frac{1}{n} \sum_{i=0}^{n-1} x_{i}, \\
G & :=\left\{q \in E: \lim _{n \rightarrow \infty} D_{g}\left(q, x_{n}\right) \text { exists }\right\}, \text { and } \\
G_{1} & :=\left\{q \in E: \text { the sequence }\left\{D_{g}\left(q, x_{n}\right)\right\}_{n \in \mathbb{N} \cup\{0\}} \text { is non-increasing }\right\} .
\end{aligned}
$$

Lemma 3. If $G_{1} \neq \varnothing$, then $G_{1}$ is closed and convex.

Proof. Let $n \geq 0$ and $G_{1, n}:=\left\{z \in E: D_{g}\left(z, x_{n+1}\right) \leq D_{g}\left(z, x_{n}\right)\right\}$. We have

$$
D_{g}\left(z, x_{n+1}\right) \leq D_{g}\left(z, x_{n}\right)
$$

if and only if

$$
g(z)-g\left(x_{n+1}\right)-\left\langle z-x_{n+1}, \nabla g\left(x_{n+1}\right)\right\rangle \leq g(z)-g\left(x_{n}\right)-\left\langle z-x_{n}, \nabla g\left(x_{n}\right)\right\rangle,
$$

if and only if

$$
\left\langle z, \nabla g\left(x_{n}\right)-\nabla g\left(x_{n+1}\right)\right\rangle \leq g\left(x_{n+1}\right)-g\left(x_{n}\right)+\left\langle x_{n}, \nabla g\left(x_{n}\right)\right\rangle-\left\langle x_{n+1}, \nabla g\left(x_{n+1}\right)\right\rangle .
$$

Clearly, all $G_{1, n}$ are closed and convex, and thus so is $G_{1}=\bigcap_{n=1}^{\infty} G_{1, n}$.

The following theorem is an extension of the corresponding one of Takahashi and Takeuchi [32].

Theorem 1. Let $E$ be a reflexive Banach space and let $g: E \rightarrow \mathbb{R}$ be a nice Bregman function. Let $\left\{x_{n}\right\}_{n \in \mathbb{N} \cup\{0\}}$ be a Bregman generalized hybrid sequence in $E$ with respect to $D_{g}$. Assume that $\left\{x_{n}\right\}_{n \in \mathbb{N} \cup\{0\}}$ is weakly asymptotically regular. Then the following are equivalent:

(i) $G_{1} \neq \varnothing$.

(ii) $G \neq \varnothing$.

(iii) $\left\{x_{n}\right\}_{n \in \mathbb{N}}$ is bounded in $E$.

(iv) $\quad\left\{x_{n}\right\}_{n \in \mathbb{N}}$ converges weakly to some $p \in E$, as $n \rightarrow \infty$.

In this case, the weak limit $p=\lim _{n \rightarrow \infty} x_{n}=\lim _{n \rightarrow \infty} s_{n} \in G_{1}$, is the Bregman asymptotic center of the sequence $\left\{x_{n}\right\}_{n \in \mathbb{N} \cup\{0\}}$ in $E$.

Proof. It is clear that (i) implies (ii). The assertion (ii) implying (iii) follows from Definition 1(ii). 
Let us show that (iv) implies (i). It is clear that the Cesáro means $s_{n} \rightarrow p$. In the light of the three-point identity (2), we have

$$
\left\langle x_{l}-p, \nabla g\left(x_{m}\right)-\nabla g(p)\right\rangle=D_{g}\left(x_{l}, p\right)+D_{g}\left(p, x_{m}\right)-D_{g}\left(x_{l}, x_{m}\right), \quad \text { for all } l, m \in \mathbb{N} .
$$

It follows

$$
\begin{aligned}
\left\langle x_{i+1}-p, \nabla g\left(x_{k+1}\right)-\nabla g(p)\right\rangle & =D_{g}\left(x_{i+1}, p\right)+D_{g}\left(p, x_{k+1}\right)-D_{g}\left(x_{i+1}, x_{k+1}\right), \\
\left\langle x_{i+1}-p, \nabla g\left(x_{k}\right)-\nabla g(p)\right\rangle & =D_{g}\left(x_{i+1}, p\right)+D_{g}\left(p, x_{k}\right)-D_{g}\left(x_{i+1}, x_{k}\right), \\
\left\langle x_{i}-p, \nabla g\left(x_{k+1}\right)-\nabla g(p)\right\rangle & =D_{g}\left(x_{i}, p\right)+D_{g}\left(p, x_{k+1}\right)-D_{g}\left(x_{i}, x_{k+1}\right), \\
\left\langle x_{i}-p, \nabla g\left(x_{k}\right)-\nabla g(p)\right\rangle & =D_{g}\left(x_{i}, p\right)+D_{g}\left(p, x_{k}\right)-D_{g}\left(x_{i}, x_{k}\right),
\end{aligned}
$$

Since $\left\{x_{n}\right\}_{n \in \mathbb{N} \cup\{0\}}$ is a Bregman generalized hybrid sequence, for some real scalars $\alpha, \beta$ we have

$$
\begin{gathered}
\alpha\left\langle x_{i+1}-p, \nabla g\left(x_{k+1}\right)-\nabla g(p)\right\rangle-\beta\left\langle x_{i+1}-p, \nabla g\left(x_{k}\right)-\nabla g(p)\right\rangle \\
\quad+(1-\alpha)\left\langle x_{i}-p, \nabla g\left(x_{k+1}\right)-\nabla g(p)\right\rangle-(1-\beta)\left\langle x_{i}-p, \nabla g\left(x_{k}\right)-\nabla g(p)\right\rangle \\
\geq(\alpha-\beta)\left(D_{g}\left(x_{i+1}, p\right)-D_{g}\left(x_{i}, p\right)\right)+D_{g}\left(p, x_{k+1}\right)-D_{g}\left(p, x_{k}\right) .
\end{gathered}
$$

Since $\left\{x_{n}\right\}_{n \in \mathbb{N} \cup\{0\}}$ is bounded, $\frac{1}{n} \sum_{i=0}^{n-1} x_{i+1}-s_{n}=\frac{x_{n}-x_{0}}{n} \longrightarrow 0$ as $n \rightarrow \infty$. Moreover, due to the local boundedness of $g$, we have $\left\{D_{g}\left(x_{n}, p\right)\right\}_{n \in \mathbb{N} \cup\{0\}}$ is bounded. Summing up (4) from $i=0$ to $i=n-1$, dividing by $n$ and letting $n \rightarrow \infty$, we get $0 \geq D_{g}\left(p, x_{k+1}\right)-D_{g}\left(p, x_{k}\right)$. This ensures that $p \in G_{1}$.

Now, we show (iii) implies (iv). By the boundedness of $\left\{x_{n}\right\}_{n \in \mathbb{N} \cup\{0\}}$, there is a weakly convergent subsequence $x_{n_{j}} \rightarrow p$ for some point $p$ in $E$. As in (4), for some real scalars $\alpha$ and $\beta$ we have

$$
\begin{aligned}
& \alpha\left\langle x_{n_{j}+i+1}-p, \nabla g\left(x_{k+1}\right)-\nabla g(p)\right\rangle-\beta\left\langle x_{n_{j}+i+1}-p, \nabla g\left(x_{k}\right)-\nabla g(p)\right\rangle \\
& \quad+(1-\alpha)\left\langle x_{n_{j}+i}-p, \nabla g\left(x_{k+1}\right)-\nabla g(p)\right\rangle-(1-\beta)\left\langle x_{n_{j}+i}-p, \nabla g\left(x_{k}\right)-\nabla g(p)\right\rangle \\
& \geq(\alpha-\beta)\left(D_{g}\left(x_{n_{j}+i+1}, p\right)-D_{g}\left(x_{n_{j}+i}, p\right)\right)+D_{g}\left(p, x_{k+1}\right)-D_{g}\left(p, x_{k}\right) .
\end{aligned}
$$

Fix a positive integer $m$. Summing up (5) from $i=0$ to $i=m-1$, dividing by $m$, letting $j \rightarrow \infty$ and using the weakly asymptotic regularity of $\left\{x_{n}\right\}_{n \in \mathbb{N}\{0\}}$, we get

$$
0 \geq(\alpha-\beta) \limsup _{j \rightarrow \infty} \frac{1}{m}\left(D_{g}\left(x_{n_{j}+m}, p\right)-D_{g}\left(x_{n_{j}}, p\right)\right)+D_{g}\left(p, x_{k+1}\right)-D_{g}\left(p, x_{k}\right) .
$$

By the local boundedness of $g$, we know that $\left\{D_{g}\left(x_{n}, p\right)\right\}_{n \in \mathbb{N} \cup\{0\}}$ is a bounded sequence. Letting $m \longrightarrow+\infty$, we get $D_{g}\left(p, x_{k+1}\right)-D_{g}\left(p, x_{k}\right) \leq 0$, which implies that $p \in G_{1}$.

Let $x_{m_{j}} \rightarrow q$ for another weak convergent subsequence. By above arguments, we have $q \in G_{1}$. Therefore,

$$
\left\langle q-p, \nabla g\left(x_{n}\right)\right\rangle=g(q)-g(p)+D_{g}\left(p, x_{n}\right)-D_{g}\left(q, x_{n}\right) \text { converges as } n \rightarrow \infty .
$$

Since $\nabla g$ is weak-to-weak ${ }^{*}$ sequentially continuous,

$$
\begin{aligned}
& \lim _{j \rightarrow \infty}\left\langle q-p, \nabla g\left(x_{n_{j}}\right)\right\rangle=\langle q-p, \nabla g(p)\rangle \\
= & \lim _{j \rightarrow \infty}\left\langle q-p, \nabla g\left(x_{m_{j}}\right)\right\rangle=\langle q-p, \nabla g(q)\rangle .
\end{aligned}
$$


It follows

$$
\langle q-p, \nabla g(q)-\nabla g(p)\rangle=0
$$

By Lemma 2(ii), we have $q=p$. This concludes that the bounded sequence $x_{n} \rightarrow p$, and thus $s_{n} \rightarrow p$.

Finally, utilizing the Bregman-Opial property, we conclude that $p$ is the Bregman asymptotic center of the sequence $\left\{x_{n}\right\}_{n \in \mathbb{N} \cup\{0\}}$ in $E$.

\section{Fixed point and Convergence Theorems}

In this section, we establish the existence of fixed points for Bregman generalized hybrid maps in $E$. This extends corresponding results in $[13,14,17,21-29,37]$. We start with the following proposition.

Proposition 2. Let $E$ be a reflexive Banach space and let $g: E \rightarrow \mathbb{R}$ be a nice Bregman function. Let $C$ be a nonempty subset of $E$ and let $T$ be a Bregman generalized hybrid self-mapping of $C$ with respect to $D_{g}$. Assume that for some $x \in C$, the sequence $\left\{x_{n}:=T^{n} x\right\}_{n \in \mathbb{N} \cup\{0\}}$ is bounded (i.e. T has a bounded orbit), and weakly asymptotically regular. Then $\left\{T^{n} x\right\}_{n \in \mathbb{N} \cup\{0\}}$ converges weakly to its Bregman asymptotic center $c$. Moreover, for every $y \in C$, the orbit $\left\{y_{n}:=T^{n} y\right\}_{n \in \mathbb{N} \cup\{0\}}$ is bounded, and the sequence $\left\{D_{g}\left(c, y_{n}\right)\right\}_{n \in \mathbb{N} \cup\{0\}}$ is non-increasing.

Proof. We first notice that both the sequences $\left\{x_{n}\right\}_{n \in \mathbb{N} \cup\{0\}}$ and $\left\{y_{n}\right\}_{n \in \mathbb{N} \cup\{0\}}$ are Bregman generalized hybrid sequences associated with the same real constants $\alpha, \beta$ from $T$. It follows from Theorem 1 that both $x_{n} \rightarrow c$ and $s_{n}=\frac{1}{n} \sum_{i=0}^{n-1} x_{i} \rightarrow c$ as $n \rightarrow \infty$, and that $c \in G_{1}$ (for the bounded sequence $\left\{x_{n}\right\}_{n \in \mathbb{N} \cup\{0\}}$ ). Let $k \geq 0$ be a fixed integer. In view of the three-point identity (2), we deduce that

$$
\begin{aligned}
& D_{g}\left(c, y_{k+1}\right) \\
= & \frac{\alpha}{n} \sum_{i=0}^{n-1} D_{g}\left(c, y_{k+1}\right)+\frac{1-\alpha}{n} \sum_{i=0}^{n-1} D_{g}\left(c, y_{k+1}\right) \\
= & \frac{\alpha}{n} \sum_{i=0}^{n-1}\left(D_{g}\left(c, x_{i+1}\right)+D_{g}\left(x_{i+1}, y_{k+1}\right)+\left\langle c-x_{i+1}, \nabla g\left(x_{i+1}\right)-\nabla g\left(y_{k+1}\right)\right\rangle\right) \\
& +\frac{1-\alpha}{n} \sum_{i=0}^{n-1}\left(D_{g}\left(c, x_{i}\right)+D_{g}\left(x_{i}, y_{k+1}\right)+\left\langle c-x_{i}, \nabla g\left(x_{i}\right)-\nabla g\left(y_{k+1}\right)\right\rangle\right) \\
= & \frac{1}{n} \sum_{i=0}^{n-1}\left(\alpha D_{g}\left(x_{i+1}, y_{k+1}\right)+(1-\alpha) D_{g}\left(x_{i}, y_{k+1}\right)\right)+\frac{1}{n} \sum_{i=0}^{n-1}\left(\alpha D_{g}\left(c, x_{i+1}\right)+(1-\alpha) D_{g}\left(c, x_{i}\right)\right) \\
& \left.+\frac{\alpha}{n} \sum_{i=0}^{n-1}\left\langle x_{i+1}-c, \nabla g\left(y_{k+1}\right)-\nabla g\left(x_{i+1}\right)\right)\right\rangle+\frac{(1-\alpha)}{n} \sum_{i=0}^{n-1}\left\langle x_{i}-c, \nabla g\left(y_{k+1}\right)-\nabla g\left(x_{i}\right)\right\rangle \\
& \frac{1}{n} \sum_{i=0}^{n-1}\left(\beta D_{g}\left(x_{i+1}, y_{k}\right)+(1-\beta) D_{g}\left(x_{i}, y_{k}\right)\right)+\frac{1}{n} \sum_{i=0}^{n-1} D_{g}\left(c, x_{i}\right) \\
& +\frac{\alpha}{n}\left(D_{g}\left(c, x_{n}\right)-D_{g}(c, x)+\left\langle x_{n}-c, \nabla g\left(y_{k+1}\right)-\nabla g\left(x_{n}\right)\right\rangle-\left\langle x-c, \nabla g\left(y_{k+1}\right)-\nabla g(x)\right\rangle\right) \\
& +\frac{1}{n} \sum_{i=0}^{n-1}\left\langle x_{i}-c, \nabla g\left(y_{k+1}\right)-\nabla g\left(x_{i}\right)\right\rangle \\
& \frac{1}{n} \sum_{i=0}^{n-1}\left(\beta D_{g}\left(x_{i+1}, y_{k}\right)+(1-\beta) D_{g}\left(x_{i}, y_{k}\right)\right)+\frac{1}{n} \sum_{i=0}^{n-1} D_{g}\left(c, x_{i}\right) \\
& -\frac{\alpha}{n}\left(D_{g}\left(x_{n}, x\right)+\left\langle x-c, \nabla g\left(y_{k+1}\right)-\nabla g(x)\right\rangle\right) \\
+ & \frac{1}{n} \sum_{i=0}^{n-1}\left\langle x_{i}-c, \nabla g\left(y_{k}\right)-\nabla g\left(x_{i}\right)\right\rangle+\frac{1}{n} \sum_{i=0}^{n-1}\left\langle x_{i}-c, \nabla g\left(y_{k+1}\right)-\nabla g\left(y_{k}\right)\right\rangle . \\
= &
\end{aligned}
$$


On the other hand, we have

$$
\frac{1}{n} \sum_{i=0}^{n-1} D_{g}\left(x_{i+1}, y_{k}\right)=\frac{1}{n} \sum_{i=0}^{n-1} D_{g}\left(x_{i}, y_{k}\right)+\frac{D_{g}\left(x_{n}, y_{k}\right)-D_{g}\left(x_{0}, y_{k}\right)}{n}
$$

Since $g$ is locally bounded, $\left\{D_{g}\left(x_{n}, y_{k}\right)\right\}$ is a bounded sequence. Thus,

$$
\frac{1}{n}\left(\sum_{i=0}^{n-1} D_{g}\left(x_{i+1}, y_{k}\right)-\sum_{i=0}^{n-1} D_{g}\left(x_{i}, y_{k}\right)\right)=\frac{D_{g}\left(x_{n}, y_{k}\right)-D_{g}\left(x_{0}, y_{k}\right)}{n} \rightarrow 0, \quad \text { as } n \rightarrow \infty .
$$

Similarly, $\left\{D_{g}\left(x_{n}, x\right)\right\}$ is a bounded sequence, and $s_{n}=\sum_{i=0}^{n} x_{n} \rightarrow c$. We see that

$$
\frac{1}{n}\left(D_{g}\left(x_{n}, x\right)+\left\langle x-c, \nabla g\left(y_{k+1}\right)-\nabla g(x)\right\rangle\right) \rightarrow 0,
$$

and

$$
\frac{1}{n} \sum_{i=0}^{n-1}\left\langle x_{i}-c, \nabla g\left(y_{k+1}\right)-\nabla g\left(y_{k}\right)\right\rangle=\left\langle s_{n}-c, \nabla g\left(y_{k+1}\right)-\nabla g\left(y_{k}\right)\right\rangle \rightarrow 0, \quad \text { as } n \rightarrow \infty .
$$

Setting

$$
\begin{aligned}
\theta_{n, k}= & \frac{\beta}{n}\left(\sum_{i=0}^{n-1} D_{g}\left(x_{i+1}, y_{k}\right)-\sum_{i=0}^{n-1} D_{g}\left(x_{i}, y_{k}\right)\right)-\frac{\alpha}{n}\left(D_{g}\left(x_{n}, x\right)+\left\langle x-c, \nabla g\left(y_{k+1}\right)-\nabla g(x)\right\rangle\right) \\
& +\frac{1}{n} \sum_{i=0}^{n-1}\left\langle x_{i}-c, \nabla g\left(y_{k+1}\right)-\nabla g\left(y_{k}\right)\right\rangle,
\end{aligned}
$$

and utilizing again the three-point identity (2), we arrive at

$$
\begin{aligned}
D_{g}\left(c, y_{k+1}\right) \leq & \frac{1}{n} \sum_{i=0}^{n-1} D_{g}\left(c, x_{i}\right)+\frac{1}{n} \sum_{i=0}^{n-1} D_{g}\left(x_{i}, y_{k}\right) \\
& \quad+\frac{1}{n} \sum_{i=0}^{n-1}\left\langle c-x_{i}, \nabla g\left(x_{i}\right)-\nabla g\left(y_{k}\right)\right\rangle+\theta_{n, k} \\
= & \frac{1}{n} \sum_{i=0}^{n-1} D_{g}\left(c, y_{k}\right)+\theta_{n, k}=D_{g}\left(c, y_{k}\right)+\theta_{n, k} .
\end{aligned}
$$

Letting $n \rightarrow \infty$, we obtain $D_{g}\left(c, y_{k+1}\right) \leq D_{g}\left(c, y_{k}\right), \forall k \geq 0$, as desired. This, together with Definition 1(ii), implies that the sequence $\left\{y_{n}\right\}_{n \in \mathbb{N} \cup\{0\}}$ is bounded.

Theorem 2. Let $E$ be a reflexive Banach space and let $g: E \rightarrow \mathbb{R}$ be a nice Bregman function. Let $C$ be a nonempty subset of $E$ and let $T$ be a Bregman generalized hybrid self-mapping of $C$ with respect to $D_{g}$. Assume that $T$ has a bounded and weakly asymptotically regular orbit $\left\{x_{n}:=T^{n} x\right\}_{n \in \mathbb{N} \cup\{0\}}$. Let $c$ be the Bregman asymptotic center of $\left\{T^{n} x\right\}_{n \in \mathbb{N} \cup\{0\}}$. Then any Bregman generalized hybrid extension $S$ of $T$ on a set containing $C \cup\{c\}$ fixing $c$, i.e., $S c=c$. 
Proof. With the three-point identity (2) and the assumption that $S$ being a Bregman generalized hybrid extension of $T$, we have

$$
\begin{aligned}
& \left\langle x_{i+1}-S c, \nabla g(S c)-\nabla g(c)\right\rangle \\
= & \alpha\left\langle x_{i+1}-S c, \nabla g(S c)-\nabla g(c)\right\rangle+(1-\alpha)\left\langle x_{i}-S c, \nabla g(S c)-\nabla g(c)\right\rangle \\
= & \alpha\left(D_{g}\left(x_{i+1}, c\right)-D_{g}\left(x_{i+1}, S c\right)-D_{g}(S c, c)\right)+(1-\alpha)\left(D_{g}\left(x_{i}, c\right)-D_{g}\left(x_{i}, S c\right)-D_{g}(S c, c)\right) \\
= & \alpha D_{g}\left(x_{i+1}, c\right)+(1-\alpha) D_{g}\left(x_{i}, c\right)-\alpha D_{g}\left(x_{i+1}, S c\right)-(1-\alpha) D_{g}\left(x_{i}, S c\right)-D_{g}(S c, c) \\
\geq & \alpha D_{g}\left(x_{i+1}, c\right)+(1-\alpha) D_{g}\left(x_{i}, c\right)-\beta D_{g}\left(x_{i+1}, c\right)-(1-\beta) D_{g}\left(x_{i}, c\right)-D_{g}(S c, c) \\
= & (\alpha-\beta)\left(D_{g}\left(x_{i+1}, c\right)-D_{g}\left(x_{i}, c\right)\right)-D_{g}(S c, c) .
\end{aligned}
$$

Summing up the above inequalities from $i=0$ to $i=n-1$, diving by $n$, letting $n \longrightarrow \infty$, and noticing that $\left(D_{g}\left(x_{n}, c\right)-D_{g}(x, c)\right) / n \rightarrow 0$ (since $g$ is locally bounded) and $s_{n} \rightarrow c$ (by Theorem 1 ), we get

$$
\langle c-S c, \nabla g(S c)-\nabla g(c)\rangle+D_{g}(S c, c) \geq 0 .
$$

This, together with (3), implies that

$$
\langle c-S c, \nabla g(S c)-\nabla g(c)\rangle-D_{g}(c, S c)+\langle c-S c, \nabla g(c)-\nabla g(S c)\rangle \geq 0,
$$

and hence $-D_{g}(c, S c) \geq 0$. This amounts to $S c=c$, and completes the proof.

Corollary 1. Let $C$ be a nonempty, closed, and convex subset of a reflexive Banach space $E$, and let $g: E \rightarrow \mathbb{R}$ be a nice Bregman function. Let $T: C \rightarrow C$ be a Bregman generalized hybrid mapping with respect to $D_{g}$. Assume that $T$ has a bounded and weakly asymptotically regular orbit $\left\{x_{n}:=T^{n} x\right\}_{n \in \mathbb{N} \cup\{0\}}$. Then the fixed point set $F(T)$ contains the Bregman asymptotic center $c$ of $\left\{T^{n} x\right\}_{n \in \mathbb{N} \cup\{0\}}$.

Proof. Note that $c$ is the weak limit of the Cesáro means $s_{n}=\frac{1}{n} \sum_{i=0}^{n-1} x_{i}$. Since $C$ is closed and convex, we know that $c \in C$. It then follows from Theorem 2 that $T c=c$.

Remark 1. Corollary 1 improves ([17], Theorem 4.3), in which it is assumed in addition that the Bregman function $g$ is uniformly convex and the orbit $\left\{T^{n} x\right\}_{n \in \mathbb{N} \cup\{0\}}$ is asymptotically regular.

In the following, we prove a fixed point theorem for Bregman generalized hybrid maps defined on non-convex domains in $E$. This is new, to the best of our knowledge, and extends or supplements the corresponding results in $[13,14,21-29,37]$.

Theorem 3. Let $E$ be a reflexive Banach space and let $g: E \rightarrow \mathbb{R}$ be a nice Bregman function. Let $T$ be a Bregman generalized hybrid self-mapping of a nonempty subset $C$ of $E$ with respect to $D_{g}$. Then $T$ has a fixed point if and only if $T$ has a bounded and weakly asymptotically regular orbit $\left\{T^{n} x\right\}_{n \in \mathbb{N}}$ of some $x \in C$, and for any $y$ in the closed convex hull $\overline{\operatorname{conv}}\left\{T^{n} x: n \geq 0\right\}$ of this orbit, there is a unique point $p \in C$ such that $D_{g}(y, p)=\inf \left\{D_{g}(y, z): z \in C\right\}$. In this case, every orbit of $T$ is bounded.

Proof. The necessity is obvious. Let us prove the sufficiency. Assume that $\left\{T^{n} x\right\}_{n \in \mathbb{N}}$ is bounded and weakly asymptotically regular for some $x \in C$. Let $c$ be the weak limit as well as the Bregman asymptotic center of $\left\{T^{n} x\right\}_{n \in \mathbb{N}}$. Since $c \in \overline{\operatorname{conv}}\left\{T^{n} x: n \geq 0\right\}$ (see Theorem 1), there exists a unique $p \in C$ such that $D_{g}(c, p) \geq D_{g}(c, z), z \in C$. From Proposition 2, we know that for every $y \in C$, the orbit $\left\{T^{n} y\right\}_{n \in \mathbb{N}}$ is bounded, and the nonnegative sequence $\left\{D_{g}\left(c, T^{n} y\right)\right\}_{n \in \mathbb{N} \cup\{0\}}$ is non-increasing. In particular, the sequence $\left\{D_{g}\left(c, T^{n} p\right)\right\}_{n \in \mathbb{N} \cup\{0\}}$ is non-increasing. Hence, we have

$$
D_{g}(c, p)=\inf \left\{D_{g}(c, z): z \in C\right\} \leq D_{g}(c, T p) \leq D_{g}(c, p) .
$$


Then, the uniqueness of $p$ implies that $T p=p$.

Definition 3. Fix a Bregman function $g: E \rightarrow \mathbb{R}$ on a reflexive Banach space $E$. We say that a nonempty subset $C$ of $E$ is Bregman Chebyshev with respect to its convex closure $\overline{\operatorname{conv}} C$, if for any $y \in \overline{\operatorname{conv}} C$, there is a unique point $x \in C$ such that $D_{g}(y, x)=\inf \left\{D_{g}(y, z) ; z \in C\right\}$.

Corollary 2. Let $E$ be a reflexive Banach space and let $g: E \rightarrow \mathbb{R}$ be a nice Bregman function. Let $C$ be a nonempty subset of $E$ which is Bregman Chebyshev with respect to its convex closure, and let $T$ be a Bregman generalized hybrid self-mapping of $C$ with respect to $D_{g}$. Then $T$ has a fixed point in $C$, if and only if, $T$ has a bounded and weakly asymptotically regular orbit $\left\{T^{n} x\right\}_{n \in \mathbb{N}}$.

Proof. This is a direct consequence of Theorem 3.

Remark 2. Our results supplement those in [21-28]. Since we do not assume the original Opial property of the underlying Banach space, as was the case in [21-28], our results are applicable in, e.g., the Lebesgue function space $L^{p}(\mu)$ setting, where $1<p<\infty$ and $p \neq 2$, while these spaces are not covered in [21-28].

\section{Bregman Absolute Fixed Points}

Recall that the set of Bregman attractive points of a map $T: C \rightarrow E$ from a nonempty subset $C$ of a Banach space $E$ is

$$
A_{g}(T):=\left\{x \in E: D_{g}(x, T y) \leq D_{g}(x, y), \forall y \in C\right\} .
$$

If $T$ is Bregman generalized hybrid, $F(T) \subseteq A_{g}(T)$. In fact, let $p \in F(T)$. By definition, for some real numbers $\alpha, \beta$ we have

$$
\alpha D_{g}(T p, T y)+(1-\alpha) D_{g}(p, T y) \leq \beta D_{g}(T p, y)+(1-\beta) D_{g}(p, y), \quad \text { for all } y \in C .
$$

Since $T p=p$, we have $D_{g}(p, T y) \leq D_{g}(p, y)$ for all $y \in C$. Thus, $p \in A_{g}(T)$.

Definition 4. Fix a Bregman function $g: E \rightarrow \mathbb{R}$ on a reflexive Banach space $E$. Let $T$ be a Bregman generalized hybrid self-mapping of a nonempty subset $C$ of $E$. A point $p \in E$ is said to be a Bregman absolute fixed point for $T$ if the extension of $T$ from $C \cup\{p\}$ to $C \cup\{p\}$ fixing $p$ is Bregman generalized hybrid, and every Bregman generalized hybrid extension of $T$ fixes $p$.

Lemma 4. Let $E$ be a reflexive Banach space and let $g: E \rightarrow \mathbb{R}$ be a nice Bregman function. Let $C$ be a nonempty subset of $E$, and $T$ be a Bregman generalized hybrid self-mapping of $C$ with respect to $D_{g}$ and corresponding constants $\alpha$ and $\beta$. Let $c$ be the Bregman asymptotic center of a bounded and weakly asymptotically regular orbit $\left\{T^{n} x\right\}_{n \in \mathbb{N}}$ of $T$. Let $S: C \cup\{c\} \longrightarrow C \cup\{c\}$ be the extension of $T$ by fixing $S c=c$.

- $\quad$ Assume $\alpha=\beta$. Then $S$ is a Bregman generalized hybrid if and only if $c \in A_{g}(T)$.

- In general, $S$ is a Bregman generalized hybrid if $c \in A_{g}(T)$ and the orbit $\left\{T^{n} z\right\}_{n \in \mathbb{N} \cup\{0\}}$ of every $z \in C$ lies on the Bregman sphere centered at $z$, with a radius of $D_{g}(z, c)$.

Proof. We first note that assuming $c \in A_{g}(T)$, the extension $S$ is a Bregman generalized hybrid self-mapping of $C \cup\{c\}$ if and only if the following inequality holds:

$$
\alpha D_{g}(T z, c)+(1-\alpha) D_{g}(z, c) \leq \beta D_{g}(T z, c)+(1-\beta) D_{g}(z, c), \quad \text { for all } z \in C .
$$

This is equivalent to $(\alpha-\beta)\left(D_{g}(z, c)-D_{g}(T z, c)\right) \geq 0$ for all $z \in C$. The assertions are now trivial.

Theorem 4. Let $E$ be a reflexive Banach space and let $g: E \rightarrow \mathbb{R}$ be a nice Bregman function. Let $C$ be a nonempty subset of $E$, and $T$ be a Bregman generalized hybrid self-mapping of $C$ with respect to $D_{g}$ and corresponding constants $\alpha$ and $\beta$. Then the Bregman asymptotic center $c$ of a bounded and weakly asymptotically 
regular orbit $\left\{T^{n} x\right\}_{n \in \mathbb{N}}$ is an absolute fixed point of $T$ if $c \in A_{g}(T)$, and either $\alpha=\beta$, or the orbit of every $x \in C$ lies on the Bregman sphere centered at $x$, with radius $D_{g}(x, c)$.

Proof. This is an immediate consequence of Theorem 2 and Lemma 4.

\section{Concluding Remarks}

In this paper, we introduce the notion of Bregman generalized hybrid sequences. Using Bregman functions and Bregman distances, we are able to prove ergodic and convergence theorems for such sequences in a reflexive Banach space, while the Bregman-Opial property plays the role of the Opial property. We also provide fixed point and absolute fixed point theorems for Bregman generalized hybrid maps defined on not necessarily convex domains in reflexive Banach spaces.

The following table summarizes the usual setups in the literature concerning the existence of a fixed point of a map $\mathbf{M}$ defined on a domain $\mathbf{D}$ of a space $\mathbf{S}$ with some extra conditions $\mathbf{E C}$, and the approximation of a fixed point by various iterative algorithms. In each column of the table, the properties stated in the above lines are stronger than those stated in the below lines.

\begin{tabular}{|c|c|c|c|}
\hline Spaces & Domains & Maps & Extra Conditions \\
\hline S1: Hilbert space & D1: convex & M1: nonexpansive & EC1: compact domain \\
\hline $\begin{array}{lrr}\text { S2: } & \text { reflexive } & \text { Banach } \\
\text { space } & \text { with } & \text { Opial }\end{array}$ & & $\begin{array}{l}\text { M2: } \\
\text { quasi-expansive }\end{array}$ & $\begin{array}{l}\text { EC2: closed and bounded } \\
\text { domain }\end{array}$ \\
\hline & & $\begin{array}{l}\text { M3: (generalized) } \\
\text { hybrid }\end{array}$ & $\begin{array}{l}\text { EC3: a bounded norm } \\
\text { asymptotically regular orbit }\end{array}$ \\
\hline $\begin{array}{l}\text { S3: reflexive Banach } \\
\text { space with a nice } \\
\text { Bregman function }\end{array}$ & D2: arbitrary & $\begin{array}{l}\text { M4: } \quad \text { Bregman } \\
\text { generalized hybrid }\end{array}$ & $\begin{array}{l}\text { EC4: a bounded weakly } \\
\text { asymptotically regular orbit }\end{array}$ \\
\hline
\end{tabular}

While the results in this paper assume the weakest conditions S3-D2-M4-EC4, those in the literature usually assume stronger conditions. Therefore, the results in this paper are among the best one would use in the current situation.

Author Contributions: Writing-original draft, E.N., L.S. and N.-C.W. All authors have made equal contributions in developing the results in this paper and preparing this manuscript. All authors have read and agreed to the published version of the manuscript.

Funding: Ngai-Ching Wong is supported partially by a Taiwan MOST grant 108-2115-M-110-004-MY2.

Acknowledgments: The authors would like to thank the referees for many helpful comments and suggestions.

Conflicts of Interest: The authors declare no conflict of interest.

\section{References}

1. Gallagher, T.M. The demiclosedness principle for mean nonexpansive maps. J. Math. Anal. Appl. 2016, 439, 832-842. [CrossRef]

2. Gallagher, T.M. A weak convergence theorem for mean nonexpansive maps. Rocky Mt. J. Math. 2017, 47, 2167-2178. [CrossRef]

3. Kang, J.I. Fixed point set of semigroups of non-expansive maps and amenability. J. Math. Anal. Appl. 2008, 341, 1445-1456. [CrossRef]

4. Opial, Z. Weak convergence of the sequence of successive approximations for nonexpansive maps. Bull. Amer. Math. Soc. 1967, 73, 595-597. [CrossRef]

5. van Dulst, D. Equivalent norms and the fixed point property for nonexpansive maps. J. Lond. Math. Soc. 1982, 25, 139-144. [CrossRef] 
6. Gossez, J.-P.; Dozo, E.L. Some geometric propertis related to the fixed point theory for nonexpansive maps. Pac. J. Math. 1972, 40, 565-573. [CrossRef]

7. Chen, G.; Teboulle, M. Convergence analysis of a proximal-like minimization algorithm using Bregman functions. SIAM J. Optim. 1993, 3, 538-543. [CrossRef]

8. Bregman, L.M. The relation method of finding the common point of convex sets and its application to the solution of problems in convex programming. USSR Comput. Math. Math. Phys. 1967, 7, 200-217. [CrossRef]

9. Censor, Y.; Lent, A. An iterative row-action method for interval convex programming. J. Optim. Theory Appl. 1981, 34, 321-358. [CrossRef]

10. Huang, Y.-Y.; Jeng, J.-C.; Kuo, T.-Y.; Hong, C.-C. Fixed point and weak convergence theorems for point-dependent $\lambda$-hybrid maps in Banach spaces. Fixed Point Theory Appl. 2011, 2011, 1-15. [CrossRef]

11. Naraghirad, E.; Wong, N.-C.; Yao, J.-C. Applications of Bregman-Opial property to Bregman nonspreading mappings in Banach spaces. Abstr. Appl. Anal. 2014, 272867. [CrossRef]

12. Chen, H.-Y.; Gehér, G.P.; Liu, C.-N.; Molnár, L.; Virosztek, D.; Wong, N.-C. Generalized isometries of the positive definite cone with respect to the quantum $\chi_{\alpha}^{2}$-divergences, Letters of Math. Physics 2017, 107, 2267-2290.

13. Kohsaka, F.; Takahashi, W. Fixed point theorems for a class of nonlinear maps related to maximal monotone operators in Banach spaces. Arch. Math. 2008, 91, 166-177. [CrossRef]

14. Kohsaka, F.; Takahashi, W. Existence and approximation of fixed points of firmly nonexpansive-type maps in Banach sapces. SIAM J. Optim. 2008, 19, 824-835. [CrossRef]

15. Takahashi, W.; Wong, N.-C.; Yao, J.-C. Fixed point theorems and convergence theorems for generalized nonspreading maps in Banach spaces. J. Fixed Point Theory Appl. 2012, 11, 159-183. [CrossRef]

16. Takahashi, W. Fixed point theorems for new nonlinear maps in a Hilbert space. J. Nonlinear Convex Anal. 2010, 11, 79-88.

17. Kuo, T.-Y.; Jeng, J.-C.; Huang, Y.-Y.; Hong, C.-C. Fixed point and weak convergence theorems for $(\alpha, \beta)$-hybrid mappings in Banach spaces. Abstr. Appl. Anal. 2012, 789043. [CrossRef]

18. Kawasaki, T. Fixed point theorems and mean convergence theorems for generalized hybrid self maps and non-self maps in Hilbert spaces. Pac. J. Optim. 2016, 12, 133-150.

19. Naraghirad, E.; Takahashi, W.; Yao, J.-C. Strong convergence of projection methods for Bregman asymptotically quasi-nonexpansive maps and equilibrium problems in Banach spaces. Pac. J. Optim. 2014, 10, 321-342.

20. Vujaković, J.; Mitrović, S.; Pavlović, M.; Radenović, S. On recent results concerning F-contraction in generalized metric spaces. Mathematics 2020, 767. [CrossRef]

21. Rouhani, B.D. Ergodic Theorems for Nonexpansive Sequences in Hilbert Spaces and Related Problems. Ph.D. Thesis, Yale University, New Haven, CT, USA, 1981.

22. Rouhani, B.D. Asymptotic behaviour of quasi-autonomous dissipative systems in Hilbert spaces. J. Math. Anal. Appl. 1990, 147, 465-476. [CrossRef]

23. Rouhani, B.D. Asymptotic behaviour of almost nonexpansive sequences in a Hilbert space. J. Math. Anal. Appl. 1990, 151, 226-235. [CrossRef]

24. Rouhani, B.D. On the fixed point property for nonexpansive maps and semigroups. Nonlinear Anal. 1997, 30, 389-396. [CrossRef]

25. Rouhani, B.D. Remarks on asymptotically nonexpansive maps in Hilbert space. Nonlinear Anal. 2002, 49, 1099-1104. [CrossRef]

26. Rouhani, B.D. Asymptotic behavior of uniformly asymptotically almost nonexpansive curves in a Hilbert space. Nonlinear Anal. 2004, 58, 143-157. [CrossRef]

27. Rouhani, B.D. Ergodic theorems for hybrid sequences in Hilbert spaces with applications. J. Math. Anal. Appl. 2014, 409, 205-2011. [CrossRef]

28. Rouhani, B.D.; Kim, J.K. Asymptotic behavior for almost-orbits of a reversible semigroup of non-Lipschitzian maps in a metric space. J. Math. Anal. Appl. 2002, 276, 422-431. [CrossRef]

29. Kocourek, P.; Takahashi, W.; Yao, J.-C. Fixed point theorems and weak convergence theorems for generalized hybrid maps in Hilbert spaces. Taiwan. J. Math. 2010, 14, 2497-2511. [CrossRef]

30. Lin, L.-J.; Takahashi, W. Attarctive point Theorems and ergodic theorems for nonlinear maps in Hilbert spaces. Taiwan. J. Math. 2012, 16, 1763-1779. [CrossRef] 
31. Lin, L.-J.; Takahashi, W.; Yu, Z.-T. Attarctive point theorems and ergodic theorems for 2-generalized nonspreading maps in Banach spaces. J. Nonlinear Convex Anal. 2013, 14, 1-20.

32. Takahashi, W.; Takeuchi, Y. Nonlinear ergodic theorem without convexity for generalized hybrid maps in a Hilbert space. J. Nonlinear Convex Anal. 2011, 12, 399-406.

33. Naraghirad, E.; Takahashi, W.; Yao, J.-C. Generalized retraction and fixed point theorems using Bregman functions in Banach spaces. J. Nonlinear Convex Anal. 2012, 13, 141-156.

34. Butnariu, D.; Iusem, A.N. Totally Convex Functions for Fixed Points Computation and Infinite Dimensional Optimization; Kluwer Academic Publishers: Dordrecht, The Netherlands, 2000.

35. Kohsaka, F.; Takahashi, W. Proximal point algorithms with Bregman functions in Banach spaces. J. Nonlinear Convex Anal. 2005, 6, 505-523.

36. Zǎlinescu, C. Convex Analysis in General Vector Spaces; World Scientific Publishing Co. Inc.: River Edge, NJ, USA, 2002.

37. Iemoto, S.; Takahashi, W. Approximating common fixed points of nonexpansive maps and nonspreading maps in a Hilbert space. Nonlinear Anal. 2009, 71, 2082-2089. [CrossRef]

(C) 2020 by the authors. Licensee MDPI, Basel, Switzerland. This article is an open access article distributed under the terms and conditions of the Creative Commons Attribution (CC BY) license (http:/ / creativecommons.org/licenses/by/4.0/). 